Supporting Information

\title{
New brush copolymers as an effective dispersant for stabilizing concentrated suspensions of silver nanoparticles
}

\author{
Tsung-Ming Chao, ${ }^{1}$ Ta-Li Hsieh, ${ }^{1}$ Shinn-Jen Chang, ${ }^{2}$ Cha-Wen Chang, ${ }^{2}$ Chia-Chen Li ${ }^{1 *}$ \\ ${ }^{1}$ Institute of Materials Science and Engineering, and Department of Materials \& Mineral \\ Resources Engineering, National Taipei University of Technology, Taipei 10608, Taiwan \\ ${ }^{2}$ Material and Chemical Research Laboratories, Industrial Technology Research Institute, Hsinchu \\ 30011, Taiwan
}

*Corresponding author. Tel.: +886-2-2771-2171 ext.2761; E-mail: ccli@mail.ntut.edu.tw

Number of pages: 10

Number of figures: 5

Number of tables: 1 


\section{Syntheses of brush copolymers}

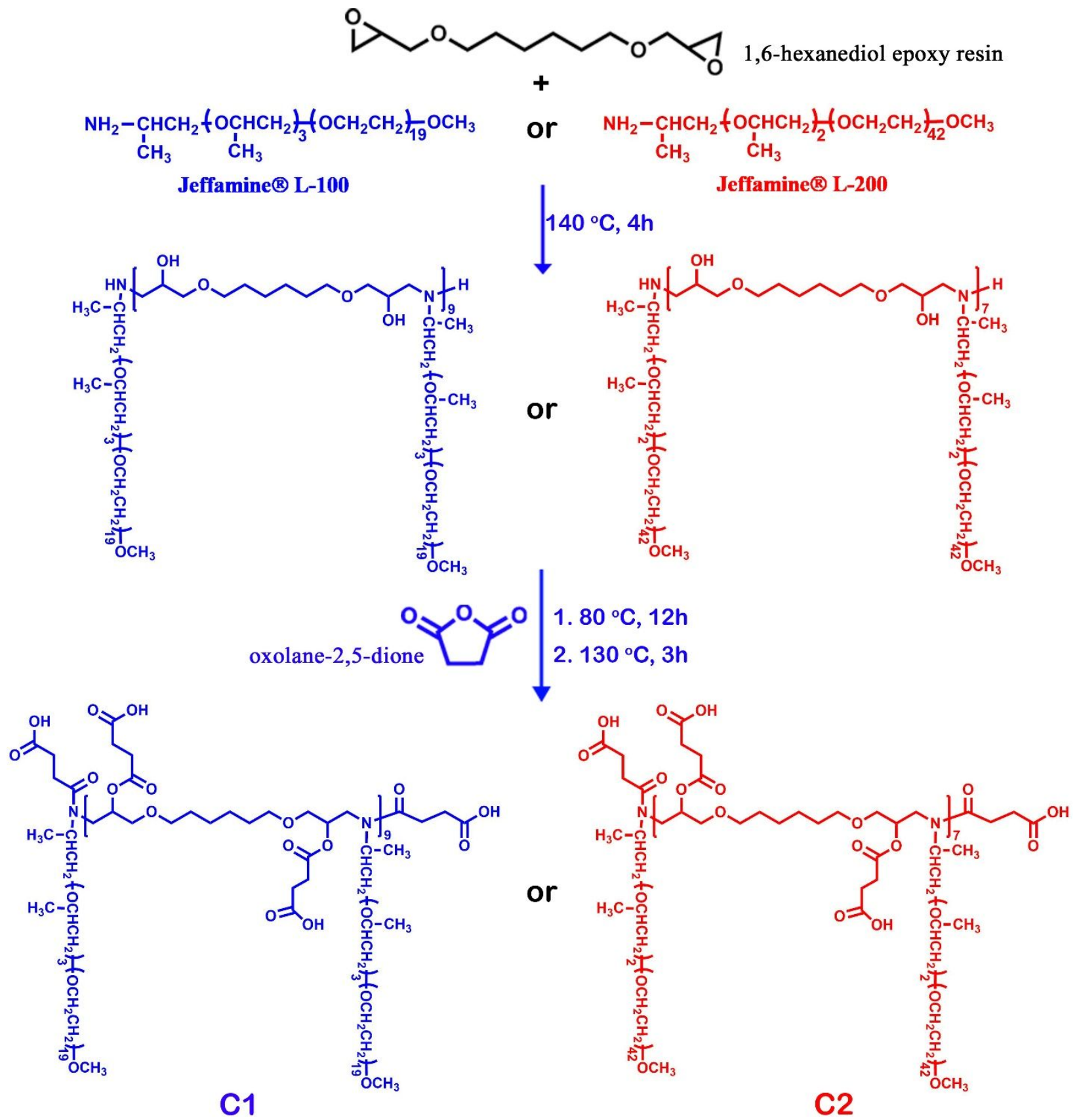

Fig. S1 Brief scheme for the syntheses of C1 and C2.

Fig. S1 shows the brief steps for $\mathrm{C} 1$ and $\mathrm{C} 2$ syntheses, in which both products have an approximated yield of $99 \%$. To the synthesis of C1, 33 mmol of Jeffamine ${ }^{\circledR L}-100$ (purchased from HUNTSMAN) and $76 \mathrm{mmol}$ of 1,6-hexanediol epoxy resin (purchased from Chang Chun 
Group) were mixed in a flask and purged with nitrogen gas for $30 \mathrm{~min}$. Then, the mixture was heated to $140^{\circ} \mathrm{C}$ to proceed with the reaction for $4 \mathrm{~h}$. Next, the reaction system was cooled down to $80{ }^{\circ} \mathrm{C}$ and added with $130 \mathrm{mmol}$ of oxolane-2,5-dione for a reaction for $12 \mathrm{~h}$. After $12 \mathrm{~h}$, the temperature was risen to $130^{\circ} \mathrm{C}$ to continue the reaction for another $3 \mathrm{~h}$, and a yellow liquid product (C1) with a yield of $99 \%$ was obtained [S1]. For the synthesis of C2, the reagent Jeffamine ${ }^{\circledR L}$ 100 was replaced by Jeffamine ${ }^{\circledR L}-200$ (also purchased from HUNTSMAN) and the reaction was conducted under the same conditions above. 


\section{NMR characterizations}
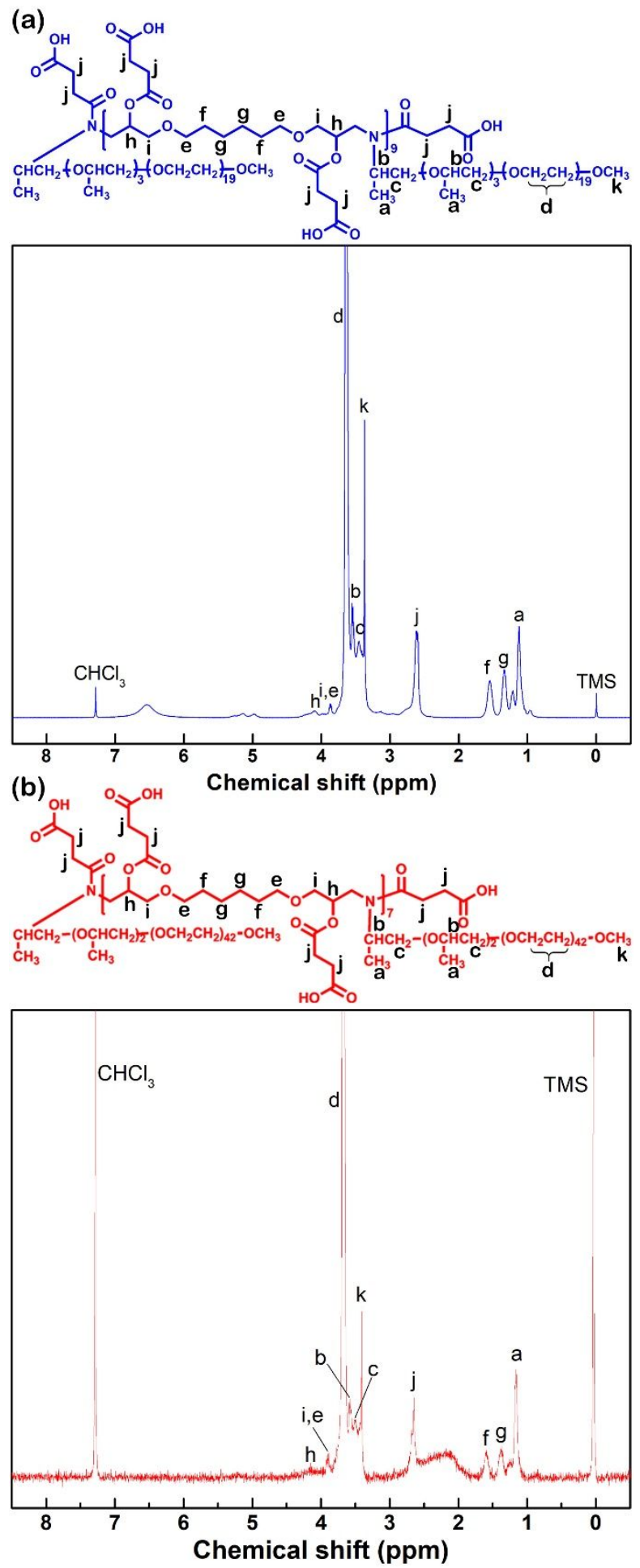

Fig. S2 NMR spectra of the synthesized (a) C1 and (b) C2, and the identifications of their chemical structures. 


\section{TGA characterization}

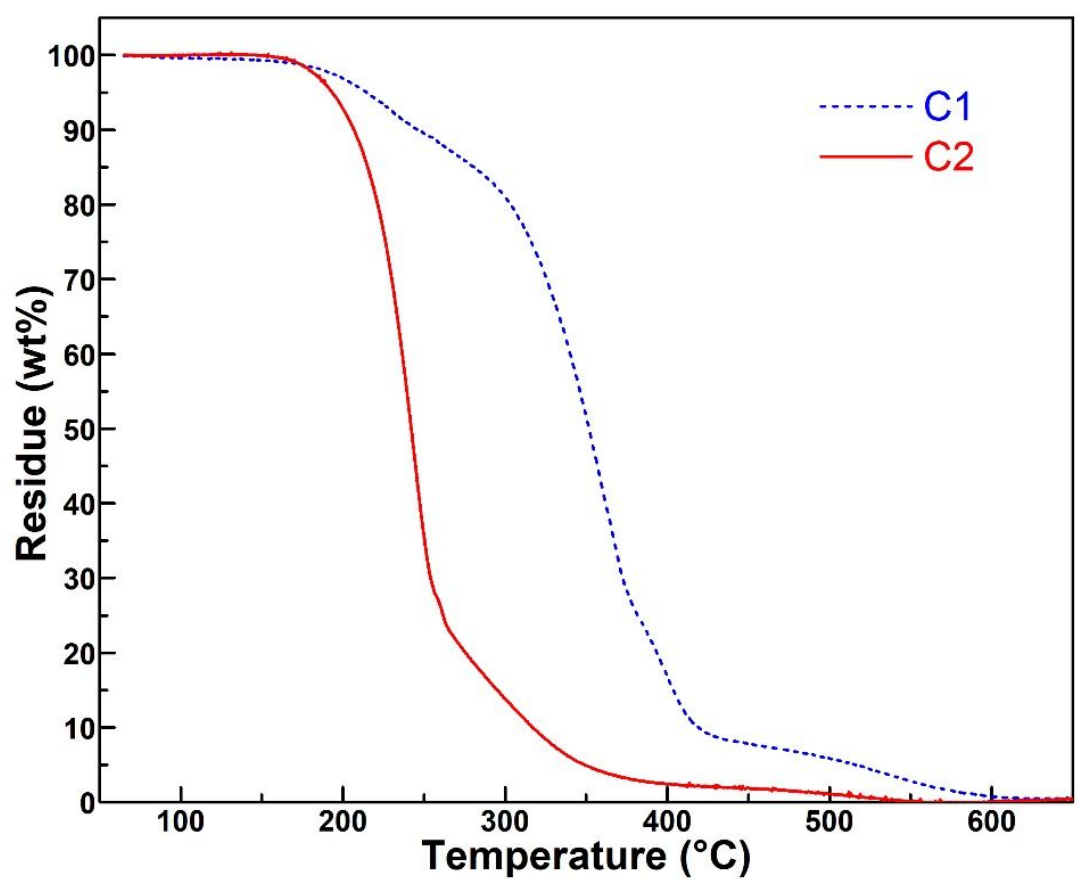

Fig. S3 TGA curves of C1 and C2.

The thermal stability of the two dispersants, $\mathrm{C} 1$ and $\mathrm{C} 2$, were measured using thermogravimetric analysis (TGA) (Q50, TA Instruments Ltd., Crawley, UK). The temperature was increased from $60{ }^{\circ} \mathrm{C}$ to $650{ }^{\circ} \mathrm{C}$ with an increasing rate of $10{ }^{\circ} \mathrm{C} \mathrm{min}-1$ under air with a flow rate of $100 \mathrm{~mL} \mathrm{~min}^{-1}$. 


\section{UV-Vis spectroscopic identification}
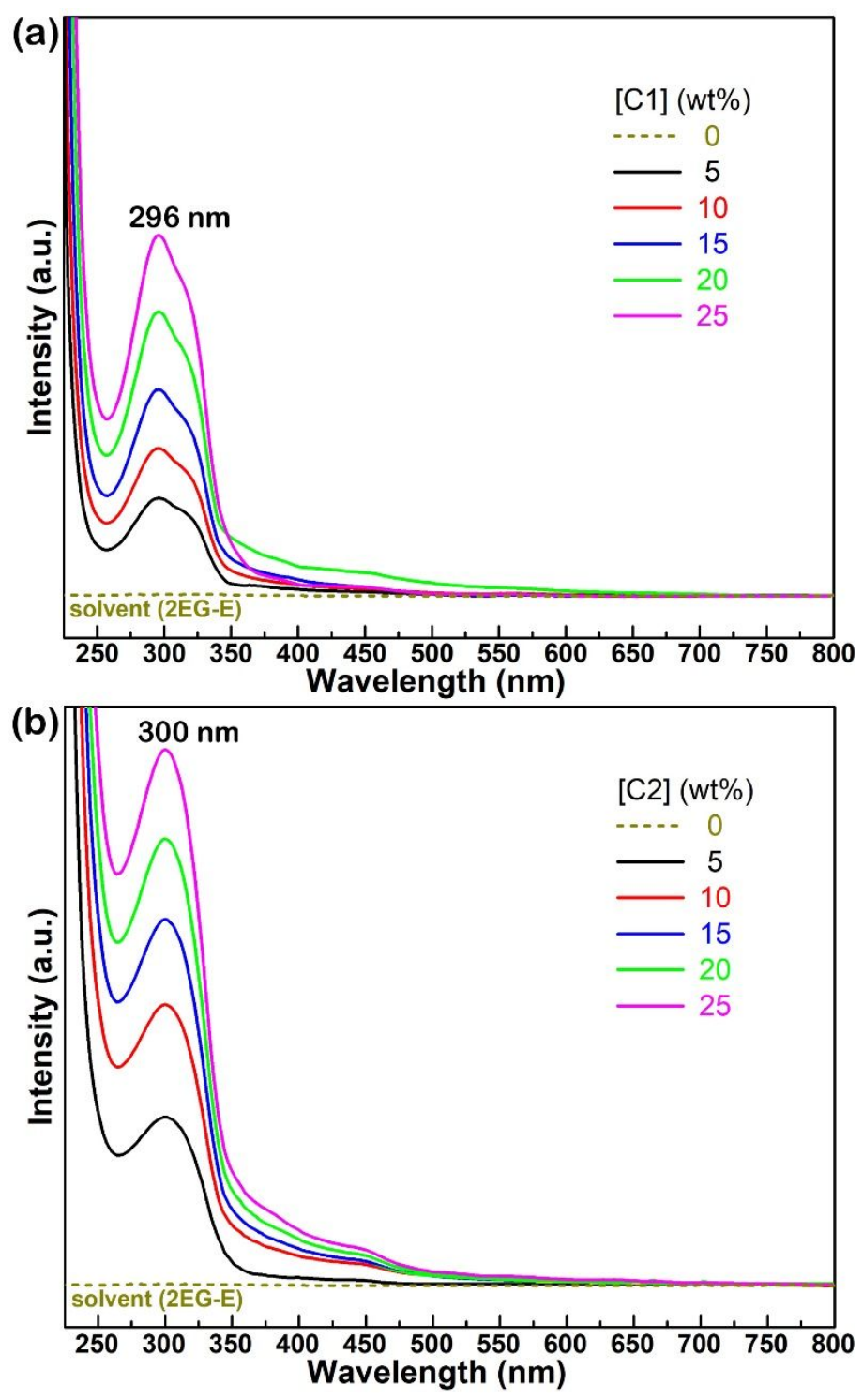

Fig. S4 UV-Vis spectra of (a) C1 and (b) C2 in 2EG-E in various concentrations.

The amount of the non-adsorbed dispersant in the supernatant was semi-quantitatively determined by ultraviolet-visible spectroscopy, in which the concentration of $\mathrm{C} 1$ and $\mathrm{C} 2$ was identified from the UV adsorption centered at 296 and $300 \mathrm{~nm}$, respectively. The data in Fig. S4 was used for calibration and determination for the concentration of the non-adsorbed dispersant. 


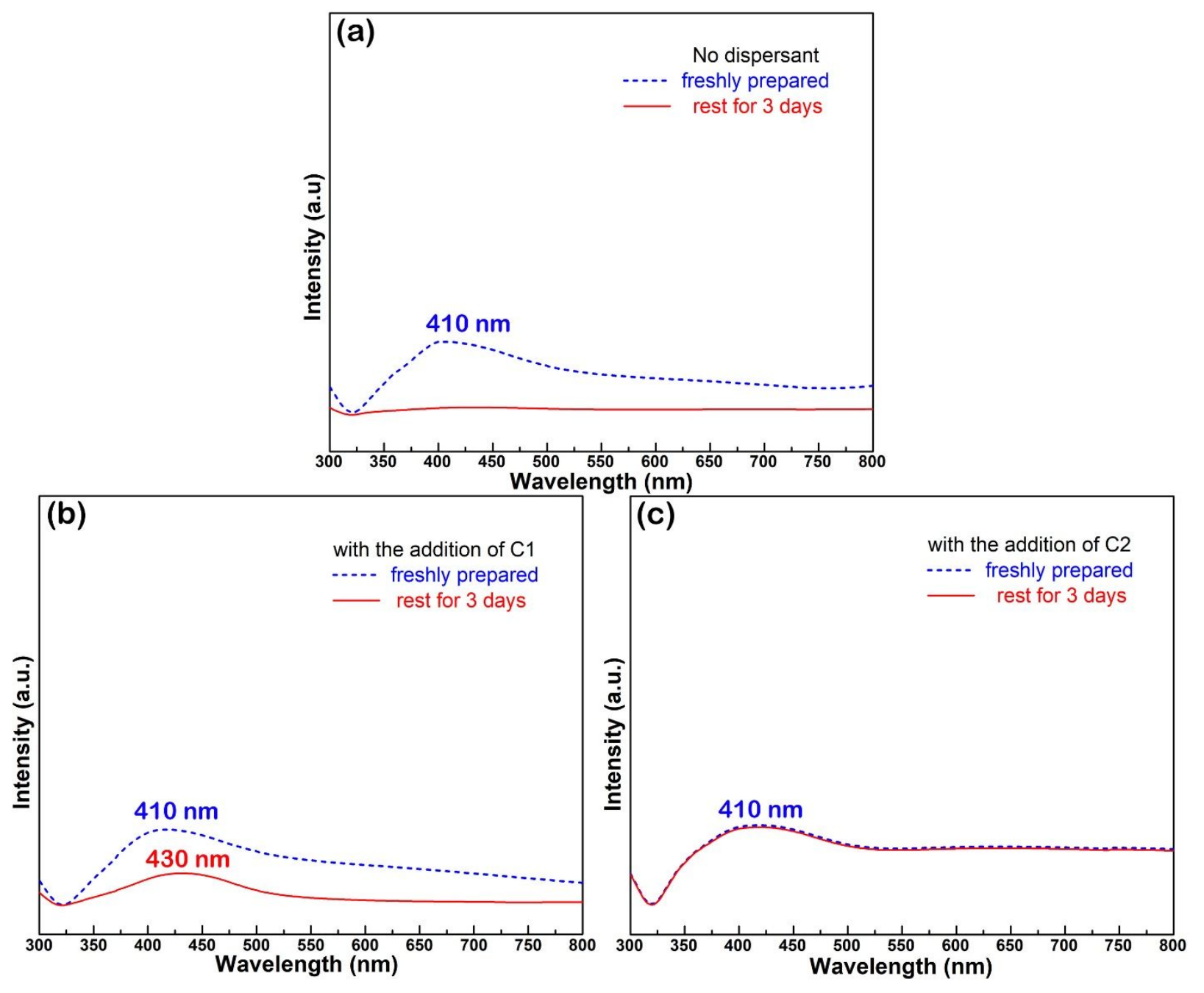

Fig. S5 UV-Vis spectra of $1 \mathrm{wt} \%$ (based upon the weight of solvent) nano-Ag suspensions (a) without added dispersant and with $5 \mathrm{wt} \%$ (based upon the weight of nano-Ag) addition of (b) $\mathrm{C} 1$ or (c) C2 after freshly prepared and 3 days.

In Fig. S5(a), the peak at $410 \mathrm{~nm}$, which should correspond to the UV adsorption of nano-Ag (particle size: $60 \mathrm{~nm}$ ), became almost disappeared after resting for 3 days. This indicates the significant settling of the nano-Ag particles. In Fig. S5(b), the peak at $410 \mathrm{~nm}$ shifted to $430 \mathrm{~nm}$ and exhibited a decreased intensity after resting for 3 days. The reduced intensity indicates the settling of the nano-Ag particles, and the red shift of the peak indicates the change in the 
morphology of the agglomerated nano-Ag. In Fig. S5(c), there is no significant change for the peak at $410 \mathrm{~nm}$, demonstrating superior dispersion stability with $\mathrm{C} 2$ addition. 


\section{Calculations for $V_{\text {sol }}$ and $\delta$}

The molecular volume of 2EG-E $\left(V_{\text {sol }}\right)$ and the $\delta$ values of $\mathrm{C} 1, \mathrm{C} 2$, and 2EG-E are not available, and hence they were approximated by theoretical calculations based on alternative group contribution method using Eqs. (S1) - (S3) [S2]:

$$
\begin{gathered}
\delta=\frac{F_{t}+B}{V}, B=277 \\
\delta=\frac{F_{t}+\frac{B}{\tilde{\tilde{n}}}}{V}, B=277 \\
V=\sum n_{i} V_{i}
\end{gathered}
$$

where $\mathrm{F}_{t}$ is a molar attraction function, $n_{i}$ is the number of each molecular segment, $V_{i}$ is the molar volume of each molecular segment, and $V$ is molecular volume. Eqs. (S1) and (S2) were used for the $\delta$ calculations of solvent (2EG-E) and polymers ( $\mathrm{C} 1$ and $\mathrm{C} 2$ ), respectively, in which $\mathrm{F}_{t}, V$, and ñ were calculated by Eqs. (S4) - (S6) using the related parameters listed in Table S1 [S2].

$$
\begin{gathered}
\mathrm{F}_{t}=\sum n_{i} F_{t, i} \\
\tilde{\mathrm{n}}=\frac{0.5}{\Delta_{T}} \\
\Delta_{T}=\sum n_{i} \Delta_{T, i}
\end{gathered}
$$

where $\Delta_{T}$ is the Lyderson correction for solvent and polymer non-ideality.

Table S1 Values of parameters for the estimation of $\delta$ [S2]

\begin{tabular}{ccccc}
\hline \multirow{2}{*}{ Structural group } & $\begin{array}{c}n_{i} \\
\mathrm{C} 1 / \mathrm{C} 2 / 2 \mathrm{EG}-\mathrm{E}\end{array}$ & $\begin{array}{c}\mathrm{F}_{t, i} \\
\left(\mathrm{~J}^{1 / 2} \mathrm{~cm}^{3 / 2} \mathrm{~mol}^{-1}\right)\end{array}$ & $\begin{array}{c}\mathrm{V}_{i} \\
\left(\mathrm{~cm}^{3} \mathrm{~mol}^{-1}\right)\end{array}$ & $\begin{array}{c}\Delta_{T, i} \\
\left(\mathrm{~J} \mathrm{~mol}^{-1}\right)\end{array}$ \\
\hline$-\mathrm{CH}_{3}$ & $50 / 32 / 1$ & 303.5 & 21.55 & 0.022 \\
$-\left(\mathrm{CH}_{2}\right)-$ & $550 / 1498 / 5$ & 269 & 15.55 & 0.020 \\
$-\mathrm{CH}-$ & $58 / 24 / 0$ & 176 & 9.56 & 0.013 \\
$-(\mathrm{C}=\mathrm{O}) \mathrm{N}-$ & $2 / 2 / 0$ & 1131 & 28.30 & 0.073 \\
$-(\mathrm{C}=\mathrm{O}) \mathrm{O}-$ & $20 / 14 / 0$ & 640 & 23.70 & 0.050 \\
$-\mathrm{N}$, tertiary & $8 / 6 / 0$ & 125 & 12.60 & 0.009 \\
$-\mathrm{O}-$, ether & $248 / 374 / 2$ & 235 & 6.45 & 0.018 \\
$-\mathrm{COOH}$ & $20 / 16 / 0$ & 565 & 26.10 & 0.039 \\
$-\mathrm{OH}$, primary & $0 / 0 / 1$ & 675 & 12.45 & 0.049 \\
\hline
\end{tabular}




\section{References}

[S1] Chang, C.W., Huang, C.M., Chang, S.J., Chen, Y.H., Teng, W.J., Tsai, S.Y., Chen, J.Y., 2016 (September 11). Patent No. TW I548673.

[S2] E.A. Grulke, Solubility parameter values, in: J. Brandrup, E.H. Immergut (Eds.), Polymer Handbook, 3rd ed., Wiley, New York, 1989, pp. VII/519-557. 